\title{
Jogos sérios brasileiros para auxilio do diagnóstico e tratamento de TDAH: revisão integrativa
}

\begin{abstract}
Brazilian serious games to help ADHD diagnosis and treatment:
\end{abstract} integrative review

Daieny Panhan Theodório ${ }^{1}$

Alessandro Pereira da Silva ${ }^{2}$

Terigi Augsuto Scardovelli ${ }^{3}$

\section{Resumo}

O TDAH é um transtorno do neurodesenvolvimento com sintomas básicos de desatenção, desorganização e/ou hiperatividade-impulsividade. Atualmente há jogos denominados jogos sérios com a finalidade de auxiliar no diagnóstico, aprendizado e tratamento de pessoas com TDAH. Objetivou-se analisar em bases de dados artigos sobre TDAH relacionados a formas de identificação de características do transtorno e formas de tratamento realizados com jogos sérios no Brasil. Especificamente: identificar quais são os jogos sérios existentes no Brasil desenvolvidos para TDAH; levantar as formas de identificação das características de TDAH com os jogos sérios; analisar as formas de tratamento existentes com a utilização de jogos sérios. Trata-se de uma revisão integrativa e para isso foram utilizadas três bases de dados, o portal de periódicos CAPES, o Google Acadêmico e o Catálogo de teses e dissertações da CAPES. Com base nos critérios de inclusão não foram selecionados artigos para análise relacionados ao tema proposto no

\footnotetext{
${ }_{1}^{1}$ Doutoranda em Engenharia Biomédica pela Universidade de Mogi das Cruzes-UMC. Psicóloga formada pela UMC, com experiência em psicologia escolar e pesquisa. Leciona no curso de Psicologia na UMC. E-mail: daienytheodorio@umc.br

2 Pós-doutorado em Engenharia Biomédica pela PUC-SP, doutor em Engenharia Biomédica, graduado em Engenharia da Computação. Atualmente, é docente e pesquisador no Programa Integrado de Engenharia Biomédica da UMC. E-mail: alessandrops@umc.br 3 Doutor em Engenharia Biomédica, graduado em Engenharia da Computação. Atualmente, é docente e pesquisador no Programa Integrado de Engenharia Biomédica da UMC. E-mail: terigiscardovelli@umc.br
} Interfaces da Educ., Paranaíba, v.11, n.32, p. 60 - 78, 2020 
portal de periódicos CAPES, foi selecionado um artigo no Google Acadêmico,' embora não esteja totalmente relacionado aos objetivos propostos e 2 arquivos no Catálogo de teses e dissertações da CAPES. Faltam artigos e jogos sérios desenvolvidos no Brasil que auxiliem o diagnóstico e terapia de pessoas com TDAH.

Palavras-chave: Jogos Sérios. TDAH. Diagnóstico. Tratamento.

\section{Abstract}

The ADHD is a neurodevelopmental disorder with basic symptoms of inattention, disorganization and/or hyperactivity-impulsivity. Nowadays there are games denominated serious games that aim to help the diagnosis, learning and treatment of people with ADHD. The objective was to analyze, in databases, ADHD articles related to ways of identifying the disorder characteristics and treatment methods performed with serious games in Brazil. Specifically: identify which are the serious games present in Brazil developed for ADHD; to bring up the ways of identifying ADHD characteristics with serious games; to analyze the existing ways of treatment using serious games. This is an integrative review and, for that, three databases were used, CAPES journal portal, Google Acadêmico and CAPES theses and dissertations catalog. Based on the inclusion criteria, no articles were selected for analysis related to the proposed theme on the CAPES journal portal, one article was selected on Google Acadêmico, although it is not completely related to the proposed goals and 2 files on the CAPES theses and dissertations catalog. There is a lack of articles and serious games developed in Brazil that help the diagnosis and therapy of people with ADHD.

Keywords: Serious Games. ADHD. Diagnosis. Treatment. 


\section{Introdução}

O Transtorno de Déficit de Atenção/Hiperatividade (TDAH) é um transtorno do neurodesenvolvimento com uma tríade sintomatológica básica: desatenção, desorganização e/ou hiperatividade-impulsividade. Quanto as duas primeiras: desatenção e desorganização estão relacionadas às características: incapacidade de permanecer em uma tarefa, parece não ouvir quando é chamado e perda de objetos constantemente. Quanto a hiperatividade-impulsividade implicam em: atividade excessiva, inquietação, incapacidade de permanecer sentado, intromissão em atividades de outros e incapacidade de aguardar a sua vez. Ressalta-se que as características observadas são mais graves do que em pessoas sem o transtorno (Manual de Diagnóstico e Estatístico de Transtornos Mentais - DSM-V publicado pela AMERICAN PSYCHIATRIC ASSOCIATION - APA, 2014; GOULARDINS et al., 2015; REINHARDT; REINHARDT, 2013).

Crianças, adolescentes e adultos com TDAH geralmente apresentam: baixa autoestima, (BIEDERMAN et al., 2012; VAQUERIZO-MADRID, 2005), maior probabilidade de envolvimento em acidentes automobilísticos (BARKLEY; COX, 2007), problemas e conflitos de relacionamentos entre familiares e amorosos, uso de substâncias ilícitas, comportamento antissocial, prática de esporte de alto risco, dificuldade em administrar finanças pessoais (BROD et al., 2012).

O TDAH é considerado um dos transtornos mais comuns na infância, com estimativa de $5 \%$ ocorrendo em crianças em idade escolar, já em adultos, a prevalência é 2,5\% (APA, 2014), ou seja, tende a diminuir, entretanto é esperado que $60 \%$ dos indivíduos adultos diagnosticados com TDAH permaneçam com o transtorno (REINHARDT; REINHARDT, 2013).

A avaliação diagnóstica para o TDAH é estritamente clínica, baseandose em critérios de classificações como o DSM-V e a Classificação Internacional de Doenças (CID) $-11^{\mathrm{a}}$ Edição (WORLD HEALTH ORGANIZATION - WHO, 2018).

As características apontadas no DSM-V são para três tipos de TDAH: combinado, predominantemente desatento e predominantemente Interfaces da Educ., Paranaíba, v.11, n.32, p. 60 - 78, 2020 
hiperativo/impulsivo. Para se estabelecer o diagnóstico é necessário que seja confirmado um padrão persistente de desatenção e/ou hiperatividadeimpulsividade, com interferência expressiva em ambientes diferentes, como: escolar, social, familiar e profissional, iniciando antes dos 12 anos e se mantendo de forma consistente (RIBEIRO et al., 2017). Na fase adulta, aspectos que diferenciam o TDAH de outras patologias são: comportamento bastante desadaptativo e uma história de vida marcada por dificuldades psicossociais, por vezes duradouras. O diagnóstico diferencial deve ser realizado e considerado outros transtornos psiquiátricos que podem interferir no diagnóstico correto: depressão maior, transtorno do humor bipolar, transtorno de ansiedade, abuso de substâncias e dependência, transtorno de personalidade Borderline e antissocial (LOPES; NASCIMENTO; BANDEIRA, 2005).

Para avaliar uma pessoa com hipótese diagnóstica de TDAH deve incluir entrevistas com a própria pessoa, com os pais ou responsáveis e professores com o intuito de investigar o desempenho escolar, é necessário também investigar as comorbidades psiquiátricas e revisão do histórico médico, psicossocial e familiar. As escalas de avaliação do comportamento preenchidas por pessoas próximas, como: pais e professores, são importantes para o processo de avaliação, mas não como único instrumento para a realização do diagnóstico (WAGNER; ROHDE; TRENTINI, 2016).

Os sintomas do TDAH estão frequentemente associados a problemas de comportamento e comorbidades (REINHARDT; REINHARDT, 2013), o reconhecimento das características é necessário para que profissionais especializados possam elaborar planos de intervenção adequados com a finalidade de aumentar a eficácia do manejo clínico e tratamento eficiente, para que a pessoa possa se relacionar em diversos ambientes sem comprometimento nos âmbitos: social, familiar, profissional e educacional (REINHARDT; REINHARDT, 2013; SIBLEY et al., 2014).

No relacionamento com o outro o que pode ser notado, é a observação de um comportamento considerado como desviante. Dependendo da frequência e/ou intensidade muito alta ou muito baixa, pode comprometer Interfaces da Educ., Paranaíba, v.11, n.32, p. 60 - 78, 2020 
as novas contingências de reforçamento que são importantes para a aprendizagem e desenvolvimento infantil (BOLSONI-SILVA; DEL-PRETTE, 2003).

As pessoas precisam se adequar aos diferentes contextos de interação social e quando não conseguem discriminar corretamente como se comportar em relação ao ambiente podem acabar tendo dificuldades em suas habilidades sociais, essas dificuldades podem ser por ineficiência ou inapropriação de comportamentos emitidos, ocasionando situações de punição.

Achenbach e Edelbrock $(1979 ; 1978)$ com base nessa perspectiva desenvolveram um modelo de classificação (atualmente utilizado pela American Psychological Association - APA) para avaliar os problemas de comportamento, em função de como eles comprometem o ambiente e a própria pessoa. Os autores classificaram como externalizantes os problemas de comportamento relacionado a agressividade e agitação são os que causam maiores impactos em familiares, já os internalizantes são prejudiciais apenas a pessoa com o problema caracterizando com retraimento social e tristeza (RIBEIRO et al., 2017).

Geralmente, a forma de tratamento é a prescrição de medicamentos estimulantes (por médicos) como o Ritalina ${ }^{\circledR}$, com o princípio ativo metilfenidato, treinos realizados por psicólogos e intervenções comportamentais por professores.

Barbosa, Peder e Silva (2016) analisaram o uso do metilfenidato em 26 crianças diagnosticadas com TDAH. Obtiveram como resultado que a maioria apresentava falta de concentração e agitação antes do diagnóstico, o que foi solucionado parcialmente após o uso do medicamento. Com o medicamento foram observadas melhoras clinicas significativas. Os professores observaram a suspeita de TDAH, comunicaram aos pais e as crianças foram diagnosticadas no início da fase pré-escolar. O uso da medicação constante traz efeitos colaterais sérios, portanto o uso deve ser ministrado quando estritamente necessário. 
Uma forma diferente de tratamento recentemente utilizada por psicólogos são os jogos sérios desenvolvidos para auxiliar no diagnóstico ou no tratamento do TDAH.

Jogos sérios são jogos projetados para educar, treinar, mudar comportamentos à medida que divertem os jogadores, embora não tenham como finalidade o entretenimento ou a diversão. A educação é o objetivo principal (ZYDA, 2005), há alguns jogos para fins educacionais (READ; SHORTELL, 2011) com a finalidade pedagógica de desenvolverem o raciocínio e o aprendizado (CORREA et al., 2008), são considerados ferramentas para o desenvolvimento integral do usuário em habilidades diversas (FALKEMBACH; GELLER; SILVEIRA, 2006). Os jogos sérios não precisam ser digitais (ANGUERA et al., 2013), porém os digitais podem ser trabalhados on-line ou sem internet (TÁRREGA et al., 2015; BAKKER et al., 2016).

David et al. (2016) fizeram uma pesquisa bibliográfica sobre a importância dos jogos sérios no desenvolvimento de novas tecnologias dedicadas à assistência médica, formas para melhorar a avaliação e o acompanhamento de diferentes patologias, auxiliando no diagnóstico e no tratamento. Apresentaram soluções tecnológicas, como sensores vestíveis, sensores ambientais (análise automática visual e de fala) e soluções digitais (jogos sérios e realidade virtual).

Taipe (2019) também desenvolveu uma pesquisa bibliográfica, investigou a respeito de modelos de jogos sérios para melhorar a atenção de crianças com TDAH e nos artigos analisados descobriu que os autores não consideram a integração de aspectos psicológicos, pedagógicos e terapêuticos que permitem o desenho e a construção de um jogo sério, visando atender às necessidades de crianças, terapeutas, pais e professores. Então, propôs um modelo que permite melhorar a atenção dos alunos que apresentam esse problema.

Frutos-Pascual et al. (2014) apresentaram uma ferramenta de teleterapia baseada em jogos sérios para a saúde, visando o aprimoramento das habilidades de gerenciamento de tempo e priorização de tarefas, indicado Interfaces da Educ., Paranaíba, v.11, n.32, p. 60 - 78, 2020 
para TDAH. Mostraram os resultados preliminares da fase piloto em um experimento realizado para avaliar o uso de tele-terapias adaptativas em um grupo de crianças em desenvolvimento típico e adolescentes com idade entre 12 e 19 anos sem TDAH.

Entre as aplicações dos jogos sérios há pesquisas sobre treinamento e utilização desses jogos em pessoas com TDAH. Para citar como exemplo, seguem alguns jogos sérios nacionais: como o jogo de Silva (2009) que desenvolveu um jogo sério para auxiliar no diagnóstico de pessoas com características de TDAH. Estudou alterações no mecanismo dopaminérgico retinal, que podem causar prejuízos na discriminação das cores ao longo da via (no espectro luminoso) azul-amarelo de pessoas com TDAH. O jogo apresenta duas versões, uma com discriminação de cores na faixa azulamarelo e a outra versão na faixa verde-vermelho. O enredo do jogo foi baseado em uma aventura de um pirata procurando um tesouro escondido em uma ilha e para seguir nesta jornada o jogador deve encontrar e interpretar as dicas espalhadas nos cenários. Durante o jogo há tarefas que precisam ser solucionadas, caso contrário não há como prosseguir par a próxima fase. Nesse jogo o jogador precisa estar atento, concentrado e memorizar a ordem das tarefas que estão no cenário em atenção dividida, concentrada e alternada.

Ianaguivara (2016) desenvolveu um jogo sério para auxiliar no diagnóstico de crianças com hipótese diagnóstica de TDAH e dificuldade no raciocínio matemático. $\mathrm{O}$ autor dividiu o jogo em tarefas que exigiam do jogador: concentração, atenção, memória, sequência de números pares, números em ordem crescente e formas geométricas.

Além disso, a implantação e inserção de jogos sérios para a psicoterapia é recente.

Com base no exposto acima objetivou-se analisar em bases de dados artigos sobre TDAH relacionados às formas de identificação de caracteristicas do transtorno e formas de tratamento realizados com jogos sérios no Brasil, especificamente pretendeu-se identificar quais são os jogos sérios existentes no Brasil desenvolvidos para TDAH, levantar as formas de Interfaces da Educ., Paranaíba, v.11, n.32, p. 60 - 78, 2020 
identificação das características de TDAH por meio dos jogos sérios e analisar as formas de tratamento existentes com a utilização de jogos sérios.

\section{Método}

Trata-se de uma pesquisa bibliográfica que pode incluir tanto publicações em jornais, livros, pesquisas e teses, como formas de comunicação oral. Seu principal objetivo é que o pesquisador entre em contato com tudo o que já foi produzido sobre o assunto em questão (MARCONI; LAKATOS, 2003). Além disso, trata-se de uma revisão integrativa, que é constituída pela integração de fatos acerca de conclusões de estudos com exatidão metodológica, que objetiva a compreensão da maneira de proceder do tema, que busca preencher uma lacuna no conhecimento (DYNIEWICZ, 2014).

Foram utilizadas para este estudo o portal de periódicos CAPES, via CAFE (Comunidade Acadêmica Federada), o Google Acadêmico e o banco de teses e dissertações da CAPES (Coordenação de Aperfeiçoamento de Pessoal de Nivel Superior).

No portal CAPES foram inseridos os descritores: "jogos sérios" AND "TDAH", sem resultados, então os descritores foram modificados para: "serious games" AND "TDAH", quando escrito por extenso Transtorno do Déficit de Atenção e Hiperatividade não foram obtidos resultados satisfatórios, ou seja, zero artigos, utilizando a sigla TDAH foi possível obter 3 artigos. Não foram selecionados filtros, como: idioma, período, tipo de material ou base de dados específica. Quando utilizados os termos: "serious games" AND "ADHD" foram obtidos 122 artigos em inglês, dessa forma não foram tabulados ou analisados, pois o objetivo deste trabalho é a investigação de artigos em português do Brasil.

No Google Acadêmico quando utilizados os descritores: jogos sérios AND TDAH, foram obtidos 63 resultados desde 2019, embora com essas palavras-chave sem a utilização de aspas os termos não são relacionados. Assim, foram utilizados os descritores: "jogos sérios" AND "TDAH" e resultou em 8 artigos no ano de 2019. 
Foi realizada também uma busca no Catálogo de teses e dissertações da CAPES com a utilização dos descritores: "jogos sérios" AND "TDAH", com as aspas o resultado são dois arquivos.

Critérios de inclusão: artigos completos sobre jogos sérios (novos ou já prontos) desenvolvidos no Brasil para pessoas com TDAH. Como o Google Acadêmico não fornece essa opção como filtro (local), foi feito download de cada um, a leitura dos resumos para a identificação se o material é nacional ou não e se foram desenvolvidos jogos para pessoas com TDAH.

\section{Resultados e discussão}

Após a busca nas três bases de dados, todo o material foi verificado antes da análise e tabulação.

Os três artigos encontrados no portal CAPES sem filtros de idioma ou período não estão relacionados aos objetivos propostos neste artigo, ou seja, não fazem referência ao TDAH relacionado aos jogos sérios.

No Catálogo de teses e dissertações da CAPES, foram inseridos os descritores: "jogos sérios" AND "TDAH", sem a utilização de quaisquer filtros, resultando em dois arquivos que seguem abaixo no Quadro 1:

Quadro 1 - Arquivos do Catálogo de teses e dissertações da CAPES

\begin{tabular}{|l|l|l|l|}
\hline \multicolumn{1}{|c|}{ Autor/ano } & \multicolumn{1}{|c|}{ Título } & \multicolumn{1}{|c|}{$\begin{array}{c}\text { Tipo de } \\
\text { material }\end{array}$} & $\begin{array}{c}\text { Relação com } \\
\text { a pesquisa }\end{array}$ \\
\hline $\begin{array}{l}\text { BASTOS, A. P. ZÃO } \\
\text { (2011) }\end{array}$ & $\begin{array}{l}\text { Utilização de um jogo sério e naive } \\
\text { bayer para auxiliar na avaliação } \\
\text { cognitiva do T. Déficit de Atenção e } \\
\text { Hiperatividade }\end{array}$ & Dissertação & Sim \\
\hline $\begin{array}{l}\text { RIVERO, T. S. } \\
\text { (2016) }\end{array}$ & $\begin{array}{l}\text { Uso de videogame como treino de } \\
\text { controle inibitório em adolescentes com } \\
\text { Transtorno do Déficit de Atenção e } \\
\text { Hiperatividade }\end{array}$ & Sim \\
\hline
\end{tabular}

Fonte: dados da pesquisa (2020)

Os dois arquivos encontrados no banco de teses e dissertações da CAPES como apresentados no Quadro 1, estão relacionados com esta pesquisa, como exemplo pode-se citar o estudo de Rivero (2016) que objetivou descrever o processo de criação de um jogo de videogame para treino de controle inibitório em adolescentes com TDAH. O autor desenvolveu um protótipo inicial do jogo e posteriormente, mais cinco Interfaces da Educ., Paranaíba, v.11, n.32, p. 60 - 78, 2020 
estudos para o refinamento das mecânicas, dinâmicas e estéticas do jogo, o protótipo foi avaliado por jogadores especialistas e foi aplicado em adolescentes com e sem TDAH.

O estudo de Bastos (2011) não apresenta o resumo da pesquisa no Catálogo de teses e dissertações da CAPES, embora há artigo posterior publicado sobre o tema em parceria.

No Google Acadêmico quando utilizados os descritores: jogos sérios AND TDAH, foram obtidos 63 resultados em 2019, sendo que 62 não estão relacionados ao tema proposto nessa busca, foram obtidos como resultados: 3 artigos de revisão de literatura sobre TDAH que não mencionam sobre jogos sérios, 14 livros com assuntos diversos, 3 artigos sobre jogos digitais, 4 sobre desenvolvimento motor, coordenação motora e psicomotricidade, 1 sobre medicalização, 3 artigos sobre dificuldade de aprendizagem, 4 sobre autismo e 1 de cada sobre os temas: serial killer, tiques, cinema e escola, violência de gênero, entre outros assuntos.

Quando utilizadas as palavras-chave: "jogos sérios" AND "TDAH", obteve-se 8 artigos em 2019, seguem descritos abaixo:

Quadro 2 - Artigos levantados no Google Acadêmico em 2019.

\begin{tabular}{|c|c|c|c|}
\hline Autor & Titulo & $\begin{array}{l}\text { Tipo de } \\
\text { material }\end{array}$ & $\begin{array}{l}\text { Relação com } \\
\text { a pesquisa }\end{array}$ \\
\hline $\begin{array}{l}\text { SILVA, J. C. P.; OLIVEIRA, E. J. } \\
\text { DE; MENDES NETO; F. M.; } \\
\text { SANTOS A. M.; QUEIROZ, P. G. } \\
\text { G.; FREITAS, A. A. C. }\end{array}$ & $\begin{array}{l}\text { Aprendizado de Máquina nos Jogos } \\
\text { para Medicina: Uma Revisão } \\
\text { Sistemática }\end{array}$ & Anais & Não \\
\hline $\begin{array}{l}\text { NASCIMENTO, L.; SANTANA, } \\
\text { B.; JUNIOR, A.; SARINHO, V. }\end{array}$ & $\begin{array}{l}\text { Treinamente: um jogo digital de } \\
\text { apoio ao desenvolvimento de } \\
\text { funções cognitivas de crianças } \\
\text { portadoras do TDAH }\end{array}$ & Anais & Sim \\
\hline $\begin{array}{l}\text { MACIEL, V. D. J.; MATIAS, B. } \\
\text { C.; SARINHO, V. T. }\end{array}$ & $\begin{array}{l}\text { Uma Revisão Sistemática da } \\
\text { Literatura de Jogos Digitais Voltados } \\
\text { para o TDAH }\end{array}$ & Anais & Não \\
\hline MARTINS, E. R. & $\begin{array}{llll}\begin{array}{l}\text { Fundamentos da } \\
\text { Computação }\end{array} & \text { Ciência da } \\
\end{array}$ & livro & Não \\
\hline SILVA, D. M.; SILVEIRA, I. F. & $\begin{array}{l}\text { As manifestações de game design } \\
\text { nas pesquisas acadêmicas que } \\
\text { relacionam os jogos digitais e o } \\
\text { ensino e a aprendizagem de } \\
\text { matemática: dez anos de estudos no } \\
\text { Brasil. }\end{array}$ & artigo & Não \\
\hline AMARAL, R. R. & $\begin{array}{l}\text { Preserve: um estudo sobre jogos } \\
\text { digitais na educação básica no }\end{array}$ & & Não \\
\hline
\end{tabular}

Interfaces da Educ., Paranaíba, v.11, n.32, p. 60 - 78, 2020 


\begin{tabular}{|l|l|l|l|}
\hline & contexto do ensino de Física. & & \\
\hline RAMOS, D. K.; GARCIA, F. A. & $\begin{array}{l}\text { Digital Games and Improvement of } \\
\text { the Inhibitory Control: a Study With } \\
\text { Children in Specialized Educational } \\
\text { Service. }\end{array}$ & Não \\
\hline MARIA, S. & & anais & Não \\
\hline
\end{tabular}

Fonte: dados da pesquisa (2020)

Como observado no Quadro 2, no Google Acadêmico foi encontrado um artigo publicado em anais de eventos, O objetivo do jogo sério desenvolvido por Nascimento et al. (2019) é auxiliar no desenvolvimento de funções de atenção e memória de forma lúdica com jogo dos erros e jogo da memória. Denominaram o jogo como Treinamente e este foi desenvolvido para computadores. Foi considerado pelos autores como um jogo educacional digital (JED) com o objetivo de auxiliar no desenvolvimento de funções cognitivas de crianças com TDAH. "Trata-se de um conjunto de mini-jogos capazes de desenvolver características como atenção, memorização, não precipitação e o foco dos respectivos jogadores" (NASCIMENTO et al., 2019, p. 5). O jogo encontrado está de acordo com os objetivos deste estudo pois relaciona os dois termos: TDAH e jogo sério, embora não forneça a identificação de características de TDAH ou auxilie no tratamento como esperado neste estudo.

Os outros materiais encontrados no Google Acadêmico não foram utilizados para análise nesta pesquisa, pois trata-se de artigos de revisão, livros, uma revista inteira de proceedigns e temas não relacionados ao TDAH.

Para o desenvolvimento desta pesquisa foram realizadas várias buscas em bases de dados diferentes com o intuito de encontrar pesquisas empíricas que relacionassem os dois temas, mesmo encontrando poucos artigos que descrevessem os resultados de jogos sérios aplicados em crianças com TDAH, foram encontrados diversos materiais que apontam pontos positivos e benefícios da utilização desses jogos. As autoras GonzálezCalleros, Guerrero-García e Navarro-Rangel (2019) podem ser citadas como exemplo, pois se referem aos jogos sérios como ferramentas que podem ser utilizadas de diversas formas e tem apresentado resultados satisfatórios em crianças com TDAH, principalmente aquelas que apresentam problemas de Interfaces da Educ., Paranaíba, v. 11, n.32, p. 60 - 78, 2020 
motivação e reagem de modo diferente do esperado em reforços positivos. Acreditam que é possivel trabalhar com jogos sérios e aprendizagem, com elementos que podem proporcionar desafios cognitivos e comportamentais e consequentemente melhorar o desempenho escolar.

Outros autores que se referem aos jogos sérios como ferramenta são Silva et al. (2017) os consideram como uma alternativa viável para auxiliar no ensino e aprendizagem de pessoas com TDAH, no diagnóstico e no tratamento, apontam que podem contribuir porque auxiliam no trabalho junto as dificuldades causadas pelo transtorno.

Oliveira, Ishitani e Cardoso (2013) assim como os autores citados acima também afirmam que os jogos sérios podem ser utilizados como instrumento de aprendizagem, diagnóstico e intervenção, acrescentam que tem aumentado a procura e o desenvolvimento desses jogos na área da saúde principalmente. Embora, mesmo com esse aumento de interesse por esses jogos na área da saúde, não há tantos jogos sérios relacionados ao TDAH, há poucas publicações a respeito do tema no Brasil, e poucos jogos desenvolvidos (OLIVEIRA; ISHITANI; CARDOSO, 2013).

Os mesmos autores supracitados continuam informando que os jogos sérios vão além do entretenimento, usando a tecnologia a favor do aprendizado. "Os jogos sérios são capazes de proporcionar ao jogador imersão, atenção, conhecimento funcional, autocontrole, tomada de decisão, autoeficácia e motivação" (OLIVEIRA; ISHITANI; CARDOSO, 2013, p. 2).

Como exemplo de jogos sérios desenvolvidos para pessoas com TDAH, pode-se descrever o estudo de Alves e Bonfim (2016) que desenvolveram o Gamebook, esse jogo pode auxiliar principalmente crianças entre 8 e 12 anos com o diagnóstico de TDAH, as autoras produziram 8 minigames, com o objetivo de "potencializar as funções executivas de memória de trabalho, planejamento, flexibilidade cognitiva, categorização, controle inibitório e atenção seletiva" (p. 9).

Peijnenborgh et al. (2016) desenvolveram um jogo denominado Timo's Adventure, que avalia atenção, planejamento, memória de trabalho, percepção de tempo e mecanismos de recompensa em crianças. Aplicaram Interfaces da Educ., Paranaíba, v.11, n.32, p. 60 - 78, 2020 
em 96 crianças com idade entre 4 e 8 anos com e sem TDAH e concluíram que o jogo foi preciso ao classificar as crianças com e sem o diagnóstico.

Robbins (2017) menciona alguns jogos sérios em seu estudo, como: o ADHD-Trainer, um jogo sério desenvolvido para "melhorar a atenção, o cálculo, o controle inibitório, a fluência verbal, a coordenação visomotora e o raciocínio perceptivo (TKT Brain Solutions SL, p. 2)”. Outro jogo comentado pelo autor é o Plan-It Commander, desenvolvido também para crianças com TDAH, "projetado para melhorar as habilidades comportamentais, como gerenciamento de tempo, habilidades sociais e memória de trabalho (PLAN-IT COMMANDER, p. 2 )”. Como último exemplo o Projeto EVO, desenvolvido pela Akili, este jogo foi desenvolvido para ativar determinadas redes neurais.

Oliveira, Lima e Couto (2019) fizeram uma revisão sobre jogos digitais e funções executivas para crianças com TDAH, concluíram que os jogos digitais não são as únicas ferramentas que professores e psicólogos possam utilizar e apontam que esses podem auxiliar a melhora no desempenho das habilidades executivas do seu funcionamento neuronal, desde que planejados e com objetivos bem definidos.

Assim como no estudo citado acima Tourinho, Bonfim e Lynn (2016) desenvolveram uma revisão sobre games, TDAH e funções executivas, relataram a dificuldade para encontrar artigos empíricos e para "identificar a contribuição dos games nas funções executivas, principalmente entre crianças com diagnóstico de TDAH” (p. 6).

"Os jogos desenvolvidos com objetivos de diagnóstico e de terapias podem ser utilizados nos ambientes escolares para melhorar o desempenho de pessoas com TDAH", auxiliando-as a desenvolver sua capacidade cognitiva. As revisões de literatura sobre o tema aprimoram o conhecimento e podem proporcionar a elevação no número de estudos sobre o tema e o desenvolvimento de jogos sérios que atendam com sucesso a demanda pedagógica e psicológica tão carente atualmente (OLIVEIRA; ISHITANI; CARDOSO, 2013, p. 7).

São jogos desafiadores que podem influenciar a motivação, concentração e inteligência de crianças com TDAH, embora para isso é Interfaces da Educ., Paranaíba, v.11, n.32, p. 60 - 78, 2020 
necessário acompanhamento e orientação de professores e psicólogos qualificados.

\section{Considerações finais}

Com base nos objetivos propostos não foi possivel encontrar nas bases dos periódicos CAPES artigos nacionais descrevendo o desenvolvimento de jogos sérios para pessoas com TDAH.

Tanto na base dos periódicos CAPES como na Google Acadêmico os descritores quando inseridos com e sem aspas, em uma tentativa de relacionar os descritores de interesse, não foram obtidos resultados satisfatórios sem as aspas. Já na base do Google Acadêmico foi encontrado um artigo relacionando os dois temas, mas não menciona a respeito de diagnóstico ou tratamento de TDAH.

No catálogo de teses e dissertações da CAPES foram encontrados dois arquivos relacionados ao tema deste estudo, porém não mencionam a respeito de diagnóstico e tratamento de pessoas com TDAH utilizando jogos sérios para esse fim.

Faltam publicações sobre jogos sérios e TDAH e há carência de jogos nacionais desenvolvidos para o diagnóstico, aprendizado e tratamento de pessoas com TDAH, pois há resultados de pesquisas que comprovam a importância e o uso desses instrumentos para essa finalidade.

Foi difícil encontrar jogos sérios nacionais relacionados ao tema TDAH, mesmo para citar como exemplo.

Faltam jogos que auxiliem pais, professores e psicólogos a identificarem as características de TDAH em crianças e jogos que auxiliem no tratamento, em treinos de atenção e concentração em intervenções psicológicas.

\section{Referências}

ACHENBACH, T. M.; EDELBROCK, C. S. The classification of child psychopathology: a review and analysis of empirical efforts. Psychol Bull, v. 85, n 6, p. 1275-1301. https://doi.org/10.1037/0033-2909.85.6.1275, 1978. 
ACHENBACH, T. M.; EDELBROCK, C. S. The Child Behavior Profile: II. Boys aged 12-16 and girls aged 6-11 and 12-16. J Consult Clin Psychol, v. 47, n 2, p. 223-33. https://doi.org/10.1037/0022-006X.47.2.223, 1979.

ALVES, L. R. G.; BONFIM, C. B. Gamebook e a estimulação de funções executivas em crianças com indicação de diagnóstico de TDAH: processo de pré-produção, produção e avaliação do software. Revista da FAEEBA Educação e Contemporaneidade, Salvador, v. 25, n. 46, p. 141-157, maio/ago. 2016.

AMERICAN PSYCHIATRIC ASSOCIATION. Manual Diagnóstico e Estatístico de Transtornos Mentais: DSM-5. 5 a .ed. Porto Alegre: Artmed, 2014.

Disponivel em: http://www.niip.com.br/wpcontent/uploads / 2018/06/Manual-Diagnosico-e-Estatistico-deTranstornos-Mentais-DSM-5-1-pdf.pdf. Acessado em: 22/08/2019.

ANGUERA, J. A.; BOCCANFUSO J; RINTOUL, J. L.; AL-HASHIMI, O.; FARAJI, F.; JANOWICH, J.; et al. Video game training enhances cognitive control in older adults. Nature, v. 501, n 7465, p. 97-101, 2013. Disponível em: https://www.researchgate.net/publication

/256448800_Video_game_training_enhances_cognitive_control_in_older_adul ts. Acessado em: 11 de janeiro de 2020.

BAKKER, D.; KAZANTZIS, N.; RICKWOOD, D.; RICKARD, N. Mental health smartphone apps: review and evidence-based recommendations for future developments. JMIR Ment Health, v. 3, n 1, p. 7, 2016. disponível em: https://mental.jmir.org/2016/1/e7/. Acessado em: 11 de janeiro de 2020.

BARBOSA, F.; PEDER, D.; SILVA, C. M. Uso de metilfenidato em crianças com transtorno de déficit de atenção e hiperatividade em um município do interior do paraná, Brasil. Acta Biomédica Brasiliensia, v. 7, n 2, dez. de 2016.

BARKLEY, R. A.; COX, D. A review of driving risks and impairments associated with attention-deficit/hyperactivity disorder and the effects of stimulant medication on driving performance. Journal of safety research, v. 38, n. 1, p. 113-128, 2007.

BASTOS, A. P. Z. Utilização de um jogo sério e naive bayer para auxiliar na avaliação cognitiva do Transtorno do Déficit de Atenção e Hiperatividade. 2011, 124 f. Mestrado em Informática. Instituição de Ensino: Universidade Federal do Estado do Rio de Janeiro, Rio de Janeiro. 2011.

BIEDERMAN, J.; SPENCER, T.; LOMEDICO, A.; DAY, H.; PETTY, C. R.; FARAONE, S. V. Deficient emotional self-regulation and pediatric attention deficit hyperactivity disorder: a family risk analysis. Psychological Medicine, v. 42, p. 639-646. 2012.

Interfaces da Educ., Paranaíba, v.11, n.32, p. 60 - 78, 2020 
BOLSONI-SILVA, A. T.; DEL PRETTE, A. Problemas de comportamento: um panorama da área. Revista Brasileira de Terapia Comportamental e Cognitiva, v. 5, n 2, p. 91-103, 2003.

BROD, M.; POHLMAN, B.; LASSER, R.; HODGKINS, P. Comparison of the burden of illness for adults with ADHD across seven countries: a qualitative study. Health and quality of life outcomes, v. 10, n. 1, p. 47, 2012.

CORRÊA, A. G. D.; ASSIS, G. A.; NASCIMENTO, M.; LOPES, R. D. GenVirtual: Um jogo musical para reabilitação de indivíduos com necessidades especiais. Revista Brasileira de Informática na Educação. v.16. n.1. p.9-17, 2008.

DAVID, R.; GROS, A.; DEUTSCH, L.; KÖNIG, A.; GUERIN, O.; ROBERT, P.; BENSAMOUN, D. Place des nouvelles technologies en neuropsychiatrie. NPG Neurologie - Psychiatrie - Gériatrie, v. 16, p. 353-357, 2016.

DYNIEWICZ, A. M. Metodologia da pesquisa em saúde para iniciantes. 3 ed. Difusão editora. São Caetano do Sul, SP, 2014.

FALKEMBACH, G. A. M.; GELLER, M.; SILVEIRA, S. R. Desenvolvimento de jogos educativos digitais utilizando a ferramenta de autoria multimídia: um estudo de caso com o ToolBook Instructor. Revista Novas Tecnologias na Educação. v.4. n.1, 2006.

FRUTOS-PASCUAL, M.; ZAPIRAIN, B. G.; ZORRILLA, A. M. Adaptive TeleTherapies Based on Serious Games for Health for People with TimeManagement and Organisational Problems: Preliminary Results. Int. J. Environ. Res. Public Health, v. 11, p. 749-772, 2014.

GONZÁLEZ-CALLEROS, C. B.; GUERRERO-GARCÍA, J.; NAVARRORANGEL, Y. Uso de juegos serios como herramienta educativa para la enseñanza a niños con tdah serious games as an educational tool to teach children suffering from adhd. BUAP-ICUAP, México, 2019.

GOULARDINS, J. B.; NASCIMENTO, R. O.; AQUINO, F. A. O.; MENDES, L. O.; CASEllA, E. B.; HASUE, R. H.; OlIVEIRA, J. A. Transtorno do Déficit de Atenção e Hiperatividade e Transtorno do Desenvolvimento da Coordenação: uma discussão das bases neurais. Rev. Neurocienc, v. 23, n 4, p. 617-624, 2015. Disponivel em:

http:/ / www.revistaneurociencias.com.br/edicoes/2015/2304/revisao/1080 revisao.pdf. Acessado em: 22/08/2019.

IANAGUIVARA, E. S. Método de concepção de serious game para avaliar conceitos básicos de matemática de alunos com falta de atenção. 2016. Tese de doutorado em engenharia biomédica. Área de concentração: 
Processamento de Sinais e Imagens Médicas. Universidade de Mogi das Cruzes, Mogi das Cruzes, 2016.

LOPES, R. M. F.; NASCIMENTO, R. F. L.; BANDEIRA, D. R. Avaliação do transtorno de déficit de atenção/ hiperatividade em adultos (TDAH): uma revisão de literatura. Avaliação Psicológica, v. 4, n. 1, p. 65-74, 2005.

MARCONI, M. A.; LAKATOS, E. M. Fundamentos da Metodologia Científica. $5^{\text {a }}$. Ed. São Paulo: Atlas, 2003.

NASCIMENTO, L.; SANTANA, B.; JUNIOR, A.; SARINHO, V. Treinamente: um jogo digital de apoio ao desenvolvimento de funções cognitivas de crianças portadoras do TDAH. In: Anais da XIX Escola Regional de Computação Bahia, Alagoas e Sergipe. SBC, 2019. p. 24-29.

PEIJNENBORGH, J. C.; HURKS, P. P. M.; ALDENKAMP, A. P.; SPEK, E. D.; RAUTERBERG, G. W. M.; VLES, J. S. H.; HENDRIKSEN, J. G. M. A study on the validity of a computer-based game to assess cognitive processes, reward mechanisms, and time perception in children aged 4-8 years. JMIR serious games, v. 4, n. 2, p. 15, 2016.

OLIVEIRA, K. S.; LIMA, C. S.; COUTO, F. P. Jogos digitais e funções executivas em escolares com transtorno do déficit de atenção e hiperatividade (TDAH): algumas reflexões. Revista Cenas Educacionais, Caetité - Bahia - Brasil, v. 2, n. 1, p. 29-43, jan./jun. 2019.

OLIVEIRA, L. B.; ISHITANI, L.; CARDOSO, A. M. Jogos computacionais e transtorno de déficit de atenção e hiperatividade: Revisão sistemática de literatura. Nuevas Ideas en Informática Educativa TISE, p. 9-11, 2013.

READ, J. L.; SHORTELL, S. M.; Interactive games to promote behavior change in prevention and treatment. J Am Med Assoc, v. 305, n. 16, p. 17045. 2011. Disponivel em: http://www.pilljogger.com/pdfs/JAMA-2011-Readjama.2011.408.pdf. Acessado em: 11 de janeiro de 2020.

REINHARDT, M. C.; REINHARDT, C. A. U. Attention deficit-hyperactivity disorder, comorbidities, and risk situations. J Pediatr, Rio de Janeiro, v. 89, n. 2, p. 124-130, 2013.

RIBEIRO, A. F.; MARINO, R. L. F.; CANTIERE, C. N.; TEIXEIRA, M. C. T. V.; ROCHA, M. M.; SCHWARTZMAN, J. S.; CARREIRO, L. L. R. Contribuição de múltiplos informantes para avaliação comportamental de adolescentes com queixas de desatenção e hiperatividade. Psico, Porto Alegre, v. 48, n. 4, p. 295-305, 2017.

RIVERO, T. S. Uso de videogame como treino de controle inibitório em adolescentes com transtorno do déficit de atenção e hiperatividade. 2016, 126 
f. Doutorado em Psicobiologia Instituição de Ensino: Universidade Federal de São Paulo, São Paulo, 2016.

ROBBINS, R. Could this be the first prescription video game? New data show it helps kids with ADHD, STAT. 2017. Disponivel em:

https://www.statnews.com/2017/12/04/ firstprescription-video-game-foradhd-from-akili/. Acessado em: 28 de janeiro de 2020.

SIBLEY, M. H.; ALTSZULER, A. R.; MORROW, A. S.; MERRILL, B. M. Mapping the academic problem behaviors of adolescents with ADHD. Sch Psychol Q. v. 29, n. 4, p. 422-437, 2014.

SILVA, A. P. Ambiente virtual para quantificação de influência de estímulos coloridos no desempenho de tarefas que exigem atenção. 2009. Tese de doutorado em engenharia biomédica. Área de concentração: Processamento de Sinais e Imagens Médicas. Universidade de Mogi das Cruzes, Mogi das Cruzes, 2009.

SILVA, L. F.; COSTA, D. B. B.; INOCÊNCIO, A. C. G. HAJED - TDAH: Heurísticas para Avaliação de Jogos Educacionais Digitais para Pessoas com TDAH. 2017. VI Congresso Brasileiro de Informática na Educação (CBIE 2017). Anais do XXIII Workshop de Informática na Escola (WIE 2017). Jataí, Goiás. 2017.

TAIPE, M. A. Modelo de serious game para mejorar la aténcion en niños con transtorno por déficit de aténcion e hiperactividad (TDAH). Risti, E17, jan. 2019.

TÁRREGA, S.; CASTRO-CARRERAS, L.; FERNÁNDEZ-ARANDA, F.; GRANERO, R.; GINERBARTOLOMÉ, C.; AYMAMÍ, N.; et al. A serious videogame as an additional therapy tool for training emotional regulation and impulsivity control in severe gambling disorder. Front Psychol, v. 6, p. 1721, 2015. Disponivel em: https://www.researchgate.net/ publication/284131374_A_Serious_Videogame_as_an_Additional_Therapy_T ool_for_Training_Emotional_Regulation_and_Impulsivity_Control_in_Severe_ Gambling_Disorder/link/566982ef08ae1a797e37538e/download. Acessado em: 11 de janeiro de 2020 .

TOURINHO, A.; BONFIM, C.; LYNN, A. Games, TDAH e funções executivas: Uma Revisão da Literatura. 2016. SBC - Proceedings of SBGames. XV SBGames - São Paulo - SP - Brazil, 2016.

VAQUERIZO-MADRID, J. Hiperactividad en el niño preescolar: descripción clinica, 40 (Supl 1), p. 25-32, 2005.

WAGNER, F.; ROHDE, L. A.; TRENTINI, C. M. Neuropsicologia do Transtorno de Déficit de Atenção/Hiperatividade: Modelos Neuropsicológicos e 
Resultados de Estudos Empíricos. Psico-USF, Bragança Paulista, v. 21, n. 3, p. 573-582, set./dez. 2016.

WORLD HEALTH ORGANIZATION. ICD 11: International Classification of Diseases 11 th revision, 2018. Disponivel em: https://icd.who.int/. Acessado em: 8 de janeiro de 2020.

ZYDA, M. Da simulação visual à realidade virtual e aos jogos. Computador, v. 38, n. 9, 2005. 